Purdue University Purdue e-Pubs

$11-1-2005$

\title{
Imaging artefacts in atomic force microscopy with carbon nanotube tips
}

\author{
Mark C. Strus \\ Purdue University, mstrus@gmail.com \\ Arvind Raman \\ Purdue University, raman@purdue.edu \\ C. V. Nguyen \\ ELORET Corp, NASA Ames Research Center
}

Follow this and additional works at: http://docs.lib.purdue.edu/nanodocs

Strus, Mark C.; Raman, Arvind ; and Nguyen, C. V., "Imaging artefacts in atomic force microscopy with carbon nanotube tips" (2005). Other Nanotechnology Publications. Paper 48.

http://docs.lib.purdue.edu/nanodocs/48

This document has been made available through Purdue e-Pubs, a service of the Purdue University Libraries. Please contact epubs@purdue.edu for additional information. 


\title{
Imaging artefacts in atomic force microscopy with carbon nanotube tips
}

\author{
M C Strus ${ }^{1}$, A Raman ${ }^{1}$, C-S Han ${ }^{2}$ and C V Nguyen ${ }^{3}$ \\ ${ }^{1}$ School of Mechanical Engineering, Purdue University, West Lafayette, IN 47907, USA \\ ${ }^{2}$ Centre for Nanomechatronics and Manufacturing, Korean Institute of Machines and \\ Materials, Taejon, Korea \\ ${ }^{3}$ ELORET Corp, NASA Ames Research Center, Moffett Field, CA 94035, USA \\ E-mail: raman@ecn.purdue.edu
}

Received 21 June 2005, in final form 28 July 2005

Published 13 September 2005

Online at stacks.iop.org/Nano/16/2482

\begin{abstract}
Dynamic atomic force microscopy (dynamic AFM) with carbon nanotube tips has been suggested as an enabling tool for high precision nanometrology of critical dimension features of semiconductor surfaces. We investigate the performance of oscillating AFM microcantilevers with multi-walled carbon nanotube (multi-walled CNT) tips interacting with high aspect ratio structures while in the attractive regime of dynamic AFM. We present experimental results on $\mathrm{SiO}_{2}$ gratings and tungsten nanorods, which show two distinct imaging artefacts, namely the formation of divots and large ringing artefacts that are inherent to CNT AFM probe operation. Through meticulous adjustment of operating parameters, the connection of these artefacts to CNT bending, adhesion, and stiction is described qualitatively and explained.
\end{abstract}

(Some figures in this article are in colour only in the electronic version)

\section{Introduction}

Single-walled and multi-walled carbon nanotubes (CNT) have been described as ideal tips for atomic force microscopy (AFM) because of their nanometre scale diameter, high length to diameter aspect ratio, and unique bending stiffness. Compared to those taken with conventional AFM tips, dynamic AFM images obtained with multi-walled carbon nanotube (MWCNT) tips (an AFM microcantilever with a CNT tip is henceforth referred to as a 'CNT AFM probe') have demonstrated improved lateral resolution of polymeric photoresist trenches on silicon [1], etched polyvinyl alcohol on silicon [2], and silacatein protein filaments [3]. MWCNT tips also offer excellent wear resistance $[4,5]$, and possess the ability to image high aspect ratio structures such as semiconductor trenches [6]. The low bending stiffness of multi-walled and single-walled carbon nanotubes allows the imaging of soft surfaces, such as biomolecules, with reduced contact forces $[7,8]$. The conductive properties of MWCNT tips also make them an ideal tool for electrical current surface mapping [9].

CNT AFM probes operated in the repulsive regime, or intermittent tapping mode, such as the single-walled carbon nanotube (SWCNT) AFM probes used by Nguyen et al [1] and Chen et al [8], exhibit tremendous lateral image resolution improvement as long as the CNT probe lightly taps the surface. Aimé et al [10] explained that single-walled nanotubes can buckle and slide during intermittent tapping mode use, causing an adhesion hysteresis that can be observed in the damping signal. The buckling of longer MWCNT tips due to repulsive forces was also investigated with the MWCNT probes tested by Lee et al [11], and the creation of undesirable image artefacts near a $30 \mathrm{~nm} \mathrm{SiO}_{2}$ grating was attributed to this buckling.

In instances when MWCNT tips are of great length ( $>1 \mu \mathrm{m}$ ) and attached to microcantilevers with stiff force constants, mechanical buckling of the CNT is often encountered and imaging artefacts are introduced. To avoid complicated responses that can arise from CNT buckling, a simple approach is to operate CNT probes in the attractive regime (also known as non-contact dynamic force microscopy (NC-DFM)), where attractive tip-sample forces are able to significantly reduce the microcantilever's vibration amplitude without the tip entering the repulsive regime. Barwich et al [12] demonstrated that microcantilevers with MWCNT tips experience reduced attractive forces, a favourable result for 


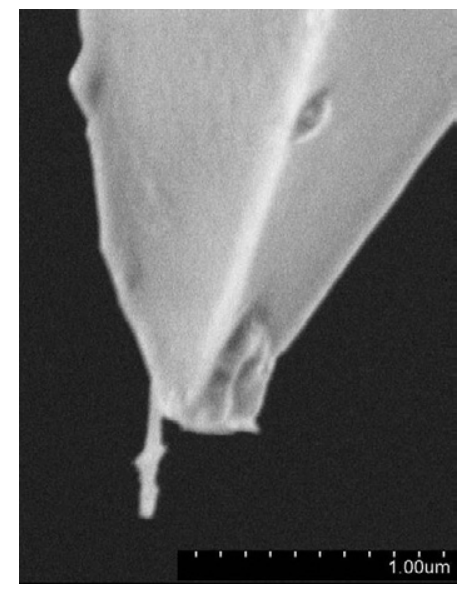

CNT AFM probe I (front)

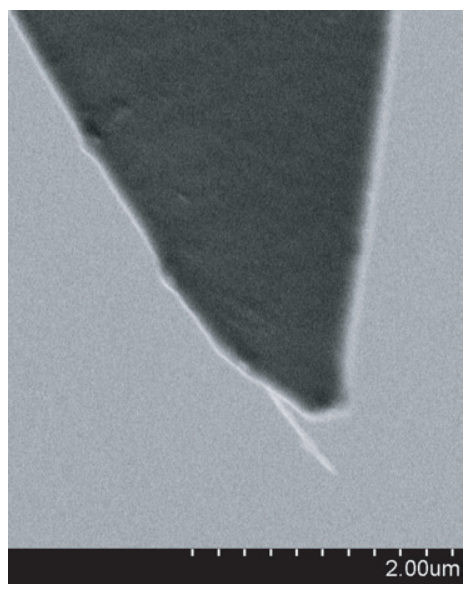

CNT AFM probe I (right)

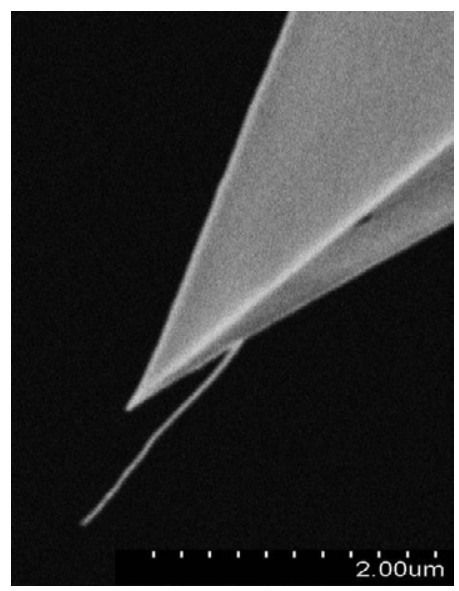

CNT AFM probe II (front)

Figure 1. FESEM images showing the front and right side images for CNT probe I and front image for CNT probe II.

Table 1. Table of properties for CNT AFM probes I and II.

\begin{tabular}{lll}
\hline CNT AFM probe & I & II \\
Microcantilever resonant frequency $(\mathrm{kHz})$ & 163 & 270 \\
Microcantilever stiffness $\left(\mathrm{N} \mathrm{m}^{-1}\right)$ & 40 & 50 \\
CNT length $(\mu \mathrm{m})$ & 0.4 & 1.2 \\
CNT diameter $(\mathrm{nm})$ & 45 & 30 \\
CNT source & CVD & CVD \\
\hline
\end{tabular}

high resolution frequency modulated attractive regime DFM. However, the analysis of Snow et al [13, 14] warned that SWCNTs tips longer than $30 \mathrm{~nm}$ could suddenly adhere to the surface during attractive regime imaging because of their low bending stiffness, and imperfect alignment angles.

While much has been reported on the improved imaging capabilities of CNT AFM probes, this paper explores undesirable image artefacts that actually degrade image resolution when using CNT AFM probes in the attractive regime. This paper builds on prior work using CNT AFM probes by Akita et al [15, 16], Kuwahara et al [17], and Park et al [18]. Through carefully designed experiments, each artefact is qualitatively linked to CNT AFM probe characteristics, such as CNT orientation, bending, stiction, and to DFM operating parameters, such as amplitude set-point ratio and driving amplitude. Experimental results help explain the origin of each imaging artefact and its dependence on the CNT probe design and DFM operation.

\section{Experimental set-up}

All experiments were performed with the Molecular Imaging ${ }^{\mathrm{TM}}$ Pico Plus atomic force microscopy system using amplitude modulation feedback for attractive mode imaging. Several MWCNT AFM probes with a variety of parameters have been tested. The CNT sources for the probes were obtained from either deposition of post-synthetic carbon nanotubes through dielectrophoresis [19] or growth of low density, individually separated MWCNTs on a Pt wire through chemical vapour deposition [20]. The CNTs were mounted on the microfabricated $\mathrm{Si}$ cantilevers and then subsequently shortened $[21,22]$. While the imaging artefacts described in this paper, namely the angle, divot, and ringing image artefacts, were observed to varying degrees for several MWCNT AFM probes, all results presented here were obtained with the two MWCNT AFM probes shown in figure 1 with properties listed in table 1.

\section{Attractive regime imaging verification using amplitude-distance curves}

A firm understanding of amplitude-distance curves when operating an amplitude modulated AFM is essential to ensure that the CNT AFM probes are operating in the attractive regime. The nature of the amplitude-distance curve for a specific CNT probe depends on two important operating parameters, namely the set-point amplitude and the set-point amplitude ratio. The set-point amplitude is the absolute value of the tip oscillation amplitude that the controller tries to keep constant while imaging the sample. The set-point amplitude ratio, on the other hand, is the ratio of the set-point amplitude to the unconstrained tip amplitude when the cantilever is far from the surface. The key to obtaining attractive regime images is minimizing the average ' $Z$-distance' between the CNT AFM probe and sample, while ensuring that the tip is not yet tapping the sample as shown in figure 2 . This is done by carefully selecting a low set-point amplitude in which attractive regime imaging can be maintained.

Figure 3 shows the amplitude-distance and phasedistance curves of CNT AFM probe I approaching a flat portion of the $\mathrm{SiO}_{2}$ surface shown in figure 4. Along the flat portion of the curve at A, no van der Waals forces are felt by the CNT tip. As the CNT AFM probe approaches the surface along A to B, the amplitude of vibration decreases due to attractive forces. When the Z-gap between the CNT AFM probe and sample is further reduced beyond the attractive region, repulsive forces come into play. In such a situation, attractive and repulsive (tapping) solutions can coexist [23]. At C, the probe amplitude and phase jump, signifying a jump from the attractive to repulsive regime. Henceforth, this will be described as the 'attractive to repulsive jump'. Upon further approach from $\mathrm{C}$ to $\mathrm{D}$, the tip experiences increased repulsive forces, which 


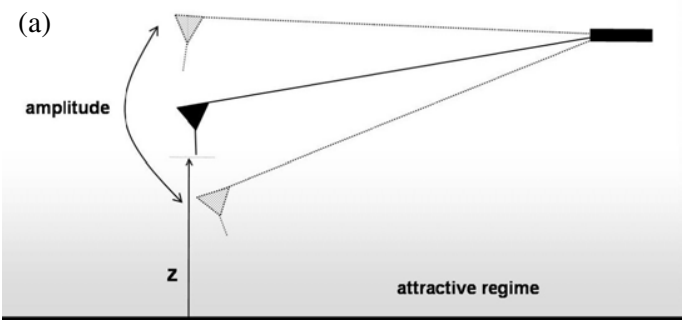

(b)

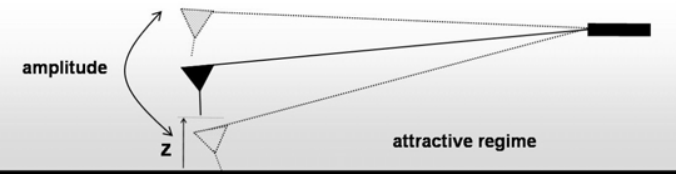

Figure 2. The $Z$-distance is the mean distance between the tip and the sample and should be minimized for attractive regime operation while ensuring the tip has not encountered the repulsive/tapping forces. The grey region qualitatively represents the region where attractive forces are felt by the tip and is usually on the order of ten nanometres. (a) Attractive regime imaging. (b) Repulsive regime imaging.
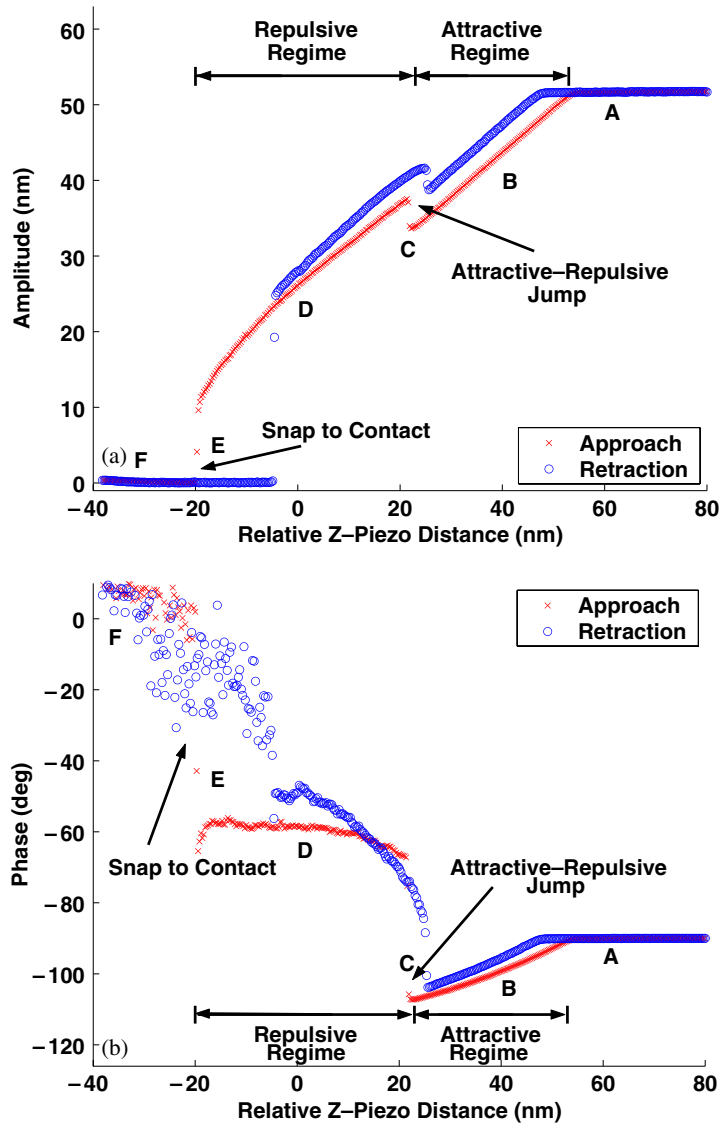

Figure 3. (a) Amplitude-distance and (b) phase-distance curves for CNT AFM probe I approaching a flat $\mathrm{SiO}_{2}$ surface. (a) Amplitude versus distance. (b) Phase versus distance.

cause a further reduction in amplitude and a simultaneous phase increase. In this repulsive region the CNT may buckle and/or slide along the surface depending on its stiffness and length $[1,10,11]$. Eventually the adhesive force between the CNT and the surface becomes large enough that the probe can no longer oscillate, as shown by the probe snap-in at E and of zero amplitude at F. In the corresponding phase-distance curve, the initial phase decrease characterizes the attractive regime along $\mathrm{B}$, while the phase jump at $\mathrm{C}$ and subsequent phase increase along $\mathrm{D}$ indicates that the probe has entered the repulsive regime. The phase again increases suddenly when the probe snaps-into the surface at E.

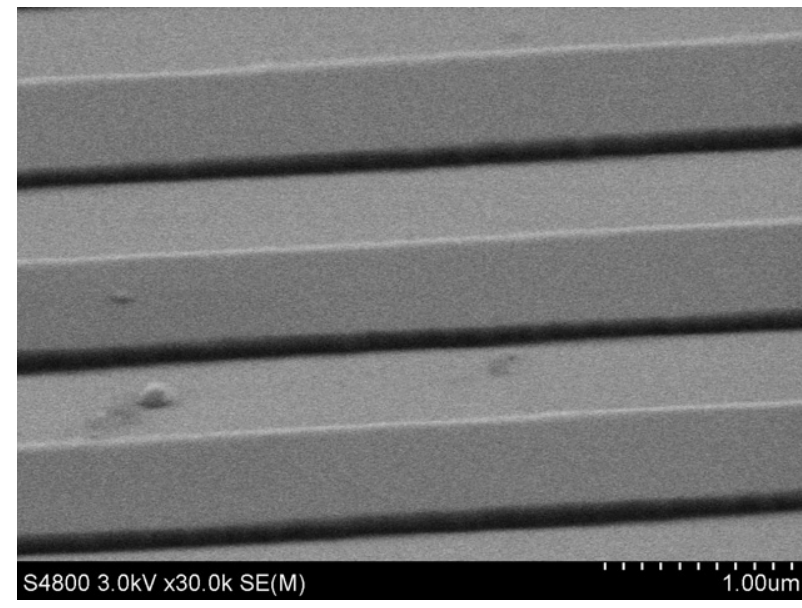

Figure 4. FESEM image showing three plateaus and almost four trenches of the $\mathrm{SiO}_{2}$ grating. Because the electron beam is focused from above, the six $100 \mathrm{~nm}$ high trench walls are difficult to resolve with the FESEM.

Our experiments show that the width of the attractive regime in figure 3 depends on a variety of parameters, namely unconstrained vibration amplitude, excitation frequency, cantilever stiffness, tip geometry, and the nature of the attractive forces. During frequency modulated DFM operation, Barwich et al [12] concluded that the smaller frequency shifts observed for CNT AFM probes compared to conventional silicon probes were due to a reduced van der Waals force between the CNT and the surface. In amplitude modulated DFM, smaller CNT tip-sample attractive forces result in a narrower width for the attractive regime, making attractive regime imaging more difficult.

The width of the non-contact region can be maximized by reducing the excitation amplitude as seen in figure 5 . When the excitation amplitude is reduced, the attractive mode imaging regime increases because the average $Z$-distance will be smaller before the probe taps the surface. As a result, attractive tip-sample forces exist during a greater percentage of the oscillation time period. Wider attractive regimes allow the user to operate in the attractive mode at lower set-point amplitude ratios and thus reduced tip-sample $Z$-distances.

Typically, only short probes exhibit a clear attractive to repulsive jump, whereas the amplitude and phase curves of longer CNTs tend to make the transition smoothly as the probe enters the repulsive regime. These differences are probably due 

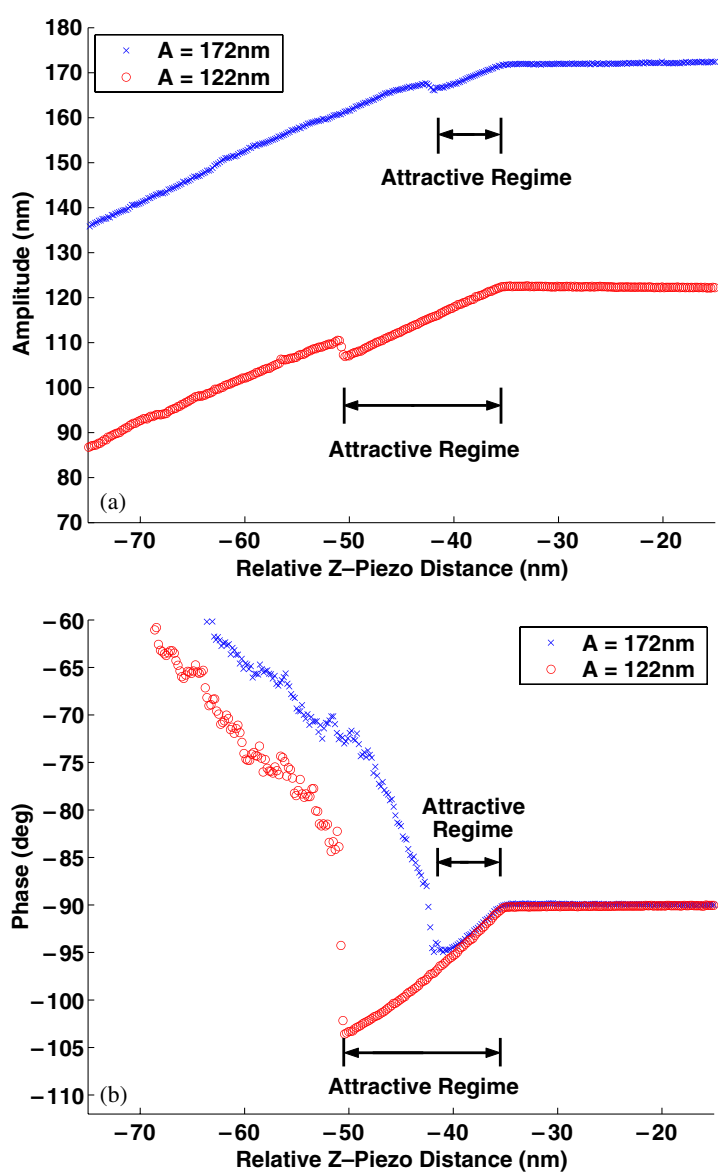

Figure 5. The (a) amplitude-distance and (b) phase-distance curves for CNT AFM probe I on a flat $\mathrm{SiO}_{2}$ surface show that lower unconstrained vibrational amplitudes are advantageous for maximizing the width of the attractive regime.

to the difference in bending stiffnesses of longer and shorter CNT tips. This in turn affects the buckling loads and the buckling mode of the CNTs on a specific surface [11].

The discussion above can be easily extended to include any additional attractive interaction forces including electrostatic forces. Consequently, the conclusions derived from the experimental results in this paper do not hinge on the sole presence of van der Waals interactions. For the specific case of the CNT tips tested on $\mathrm{SiO}_{2}$ gratings in this paper, the amplitude-distance curves did not show significant long range electrostatic forces.

It should be noted that van der Waals forces are expected to dominate the attractive tip-sample interaction, though electrostatic forces may also play a role. Nonetheless, the experimental results presented are applicable no matter what the underlying nature of the attractive tip-sample interaction is.

In what follows we will discuss in sequence how amplitude modulated AFM imaging artefacts are linked to CNT orientation, and caused by CNT bending and stiction. The DFM operating parameters are systematically changed to allow us to gain an understanding of the underlying cause of each artefact.

\section{Effect of CNT orientation angle on high resolution imaging}

The orientation angle of the attached CNT relative to the AFM microcantilever is critical for high resolution imaging. The two-dimensional profile scans of the two different edges of the $100 \mathrm{~nm}$ tall $\mathrm{SiO}_{2}$ grating in figure 6(a) show the image distortion caused by the CNT orientation angle when scanning in a single direction. Both the left and the right trench wall were scanned with CNT probe I operating in the attractive regime, with an unconstrained vibrational amplitude of $65 \mathrm{~nm}$ and a set-point amplitude ratio of $80 \%$. As the schematic diagram of figure 6(b) shows, the CNT orientation angle relative to each trench wall causes an added distortion on the right edge (RE). The measured angle of the right edge angle is about $15^{\circ}$ larger than the left edge's measured angle, while the CNT orientation angle calculated from the field emission scanning electron microscope (FESEM) image of figure 1 was $20^{\circ}$. When scanning the left edge, the free end of the CNT encounters interaction forces from the wall first, but when scanning the right edge, portions of the CNT closer to its attachment point actually encounter the trench wall before the free end of the CNT does, thus decreasing the resolution of the scan. Schlaf et al [24] demonstrated the effect of CNT alignment by scanning a linewidth at varying CNT attachment angle relative to the scan direction. The orientation of CNT
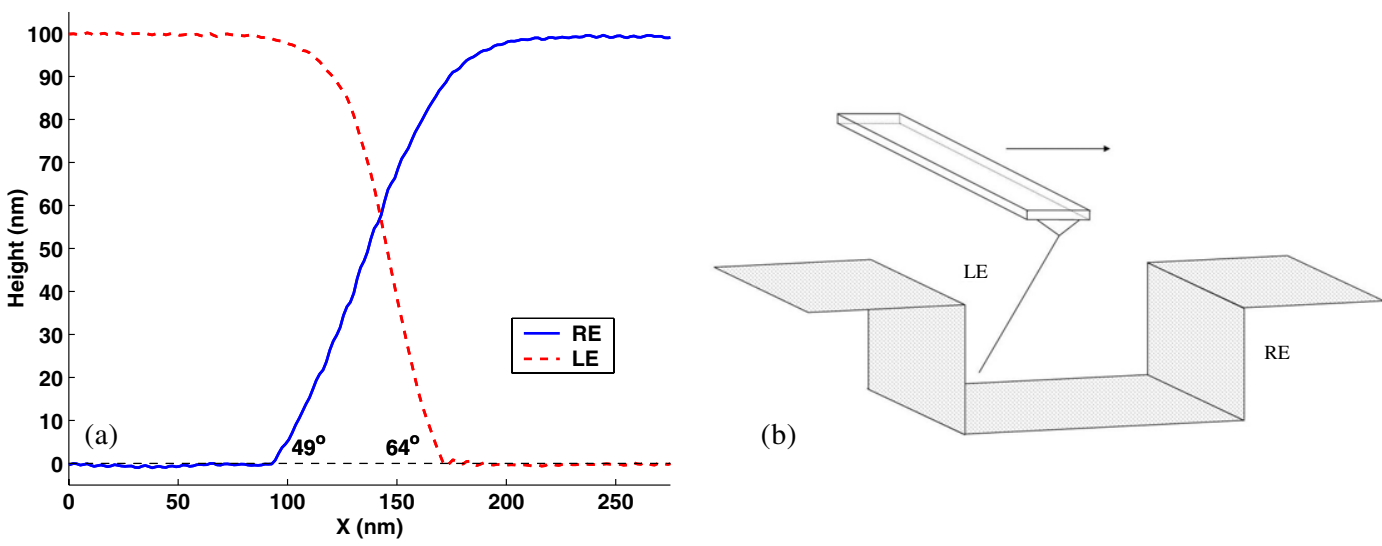

Figure 6. Two-dimensional profile scan of the right and left edges of a silicon grating overlaid to allow comparison and a schematic diagram showing the CNT orientation angle with respect to each trench wall. 

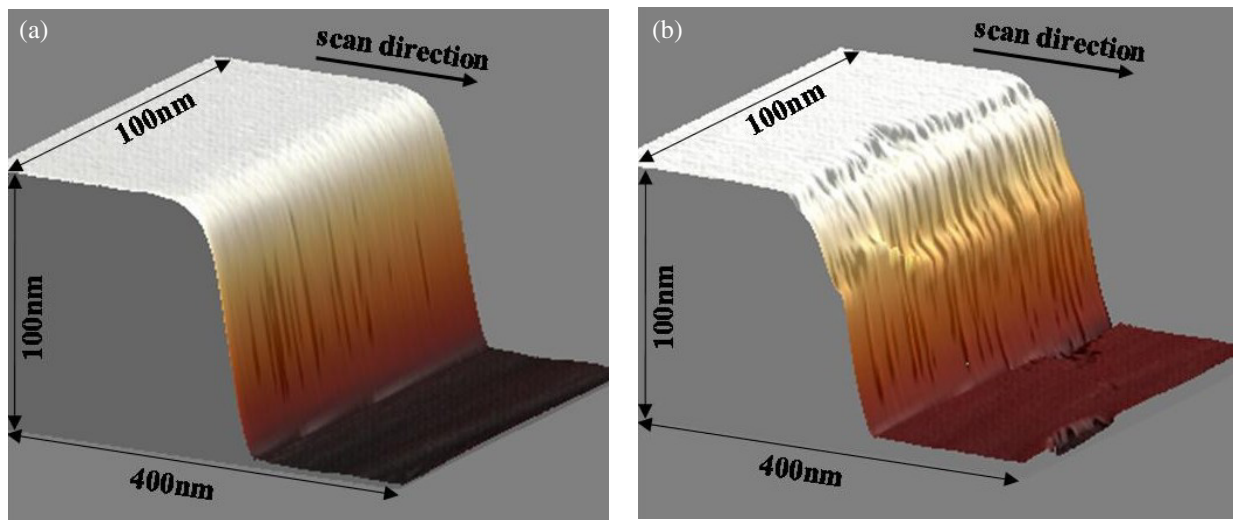

Figure 7. While scanning with CNT probe I, divot artefacts appear at the top corner of a $100 \mathrm{~nm}$ tall $\mathrm{SiO}_{2}$ grating when the set-point amplitude ratio is lowered from $91 \%$ (a) to $73 \%$ (b). The unconstrained vibrational amplitude was kept at a constant $69 \mathrm{~nm}$. (a) Undistorted image. (b) Image with divot artefacts.
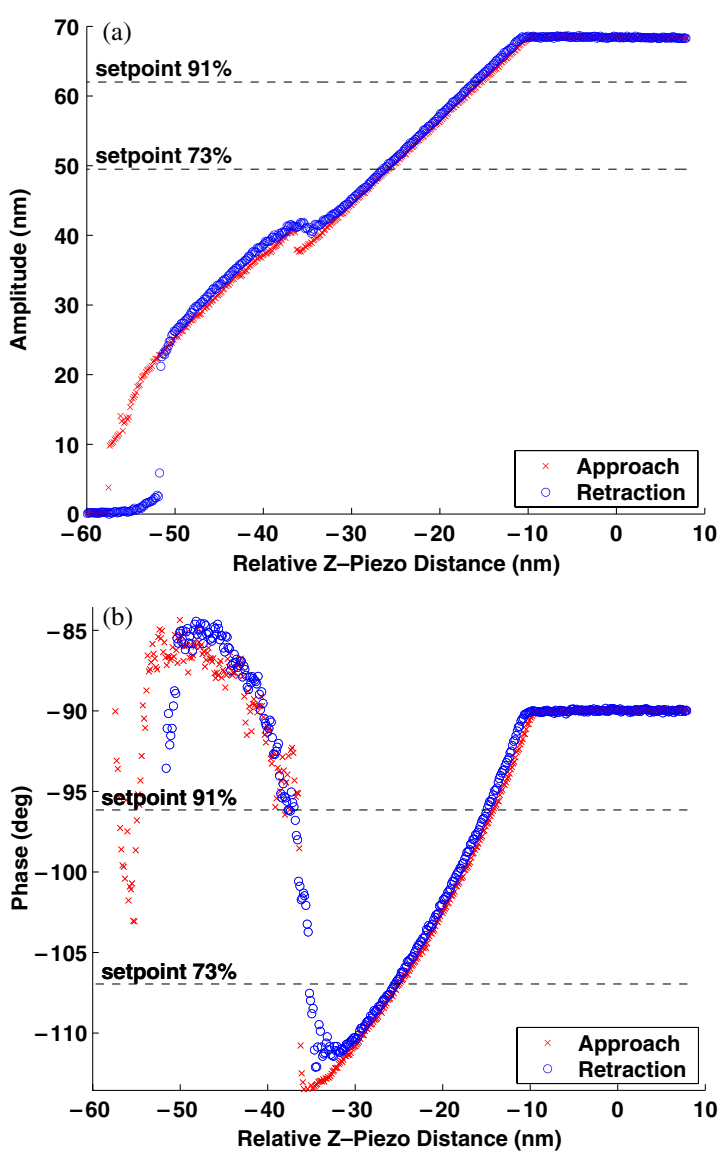

Figure 8. (a) Amplitude-distance and (b) phase-distance curves for CNT probe I show the set-point amplitude ratios chosen before obtaining the images of figure 7. The unconstrained vibration amplitude was kept at a constant $69 \mathrm{~nm}$.

tips plays a key role in the ringing artefact that will be described later.

\section{Description of divot artefact}

We now turn our attention to distinct imaging artefacts termed 'divot' artefacts that are particular to CNT AFM probe operation in the attractive regime when scanning high aspect ratio features. Figure 7 illustrates the divot artefact near the top corner of a $100 \mathrm{~nm}$ tall $\mathrm{SiO}_{2}$ trench wall obtained while using CNT probe I. In figures 7(a) and (b) the piezo-excitation amplitudes are identical as are the unconstrained vibration amplitudes of the microcantilever. However, figure 7(a) is imaged with a $91 \%$ set-point amplitude ratio while figure 7(b) is imaged with a $73 \%$ set-point amplitude ratio. To better understand the significance of the chosen set-point amplitude ratios, consider the dynamic amplitude-distance and phasedistance curves (figure 8) measured for this probe with the same excitation amplitude as was used for the images in figure 7. These amplitude-distance and phase-distance curves are measured at a point that is located far from the trench wall. Note that the $73 \%$ set-point amplitude ratio puts the probe operation very close to the attractive to repulsive jump, where there exist attractive and repulsive oscillation states with the same amplitude but different $Z$-distances. The relevance of this observation will be further explored in the ensuing paragraphs.

When operating with lower set-point amplitude ratios, the CNT AFM probe is more likely to switch from the attractive to the repulsive regime imaging whenever the vertical attractive tip-sample forces are weakest. This sudden switch from the attractive to the repulsive regime is confirmed by the topography and phase line scans in figure 9 which show a large sudden phase increase at the exact position where the divot artefact occurs in the topographic profile. This sudden phase increase, which is not seen in the undistorted profile, occurs when the CNT probe has switched from the attractive to the repulsive regime. Because the AFM controller maintains a constant vibration amplitude by adjusting the $Z$-distance, a switch from the repulsive to the attractive regime is possible near the attractive to repulsive jump. Though the amplitude is identical when switching from the attractive to the repulsive regime, the phase is much larger in the repulsive regime.

The divots occur at the top edge of the $\mathrm{SiO}_{2}$ trench wall where the vertical attractive tip-sample forces are the weakest. Where the attractive forces are especially weak, the corresponding attractive regime for the amplitude-distance curve is narrower. Thus, probes operating with very small setpoint amplitude ratios in the attractive regime may suddenly 

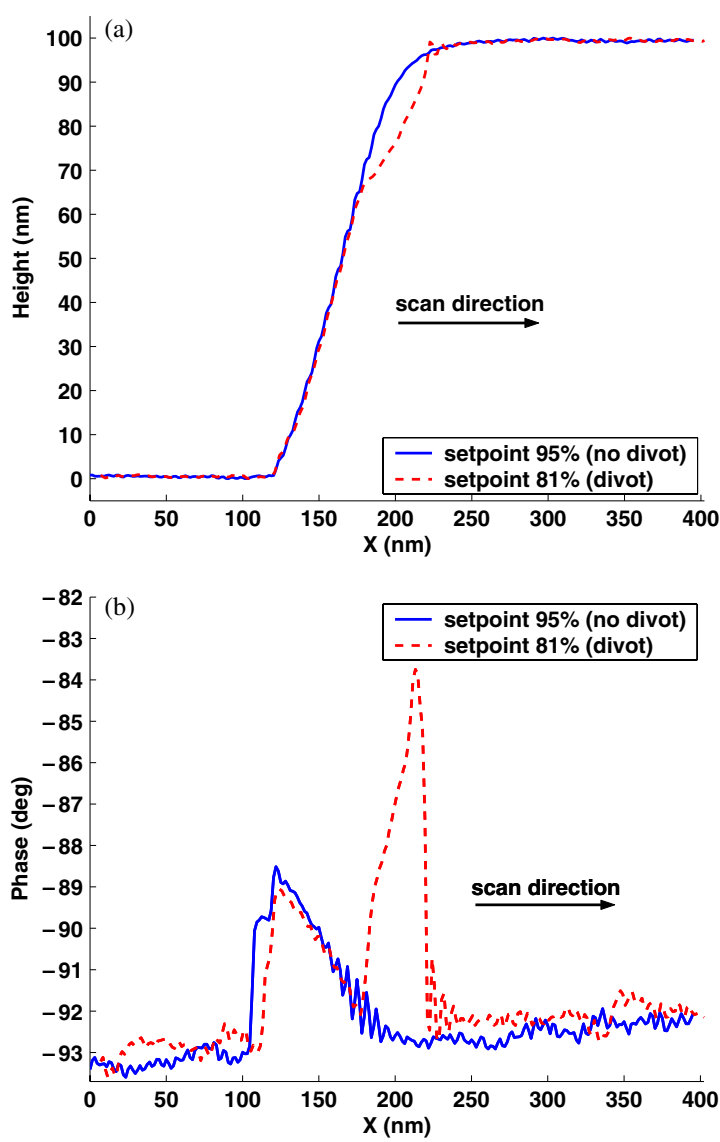

Figure 9. The (a) topography and (b) phase scan profiles for CNT probe I show a phase jump when the divot occurs, which means that the CNT probe started operating in the repulsive regime. The divot-free scan and divotted profiles were both scanned with CNT probe I with respective unconstrained amplitudes of $69 \mathrm{~nm}$ while reducing the set-point amplitude ratio from $95 \%$ to $81 \%$.

switch to repulsive regime imaging. This problem is inherent to CNT tips operating in the attractive regime because of their high aspect ratios and small tip diameters, whereas conventional AFM probes have wider conical tips that maintain sufficient vertical attractive tip-sample forces even at sharp edges. Any switch from the attractive regime to the repulsive regime must include a sudden reduction in the $Z$-distance as dictated by the amplitude-distance and phase-distance curves in figure 3. The reduction in $Z$-distance is what creates the divot.

For large excitation amplitudes, divot artefacts are unavoidable in attractive regime imaging, no matter how high the set-point amplitude ratio is chosen, as shown by the amplitude-distance curve and divotted $\mathrm{SiO}_{2}$ trench wall image in figure 10. Larger driving amplitudes result in smaller attractive regimes, in general, which in turn make the CNT AFM probe more susceptible to a switch into the repulsive regime at the $\mathrm{SiO}_{2}$ trench wall corner.

Divot artefacts can occur whenever the operator chooses a set-point corresponding to the attractive to repulsive jump, as Kuhle et al [25] reported with conventional AFM probes on a $\mathrm{SiO}_{x}$ surface. Divots occur because two different tipsample $Z$-distances lead to the same probe amplitude. In an amplitude modulated AFM scheme this leads to uncertainty about which $Z$-distance to choose to maintain a constant probe amplitude. The smaller CNT tip-sample attractive forces make CNT probes prone to divots even while operating above the attractive to repulsive jump. This makes divot artefacts unavoidable near sharp corners especially when operating with large driving amplitudes.

\section{Description of ringing artefact}

Although the high aspect ratio of the CNT AFM probes makes them especially susceptible to divots during attractive mode imaging, divot artefacts have also been shown to exist with conventional AFM probes. On the other hand, the ringing artefact caused by CNT AFM probes is not usually observed with conventional probes. Figure 11 shows the ringing artefacts observed with CNT AFM probes I and II

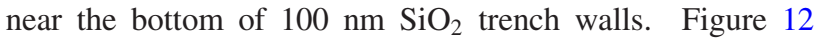
demonstrates that the ringing artefacts were only observed when the unconstrained vibration amplitude of the cantilever was reduced below a threshold value. While the set-point amplitude ratio was kept constant at $90 \%$, the ringing artefact was captured when the unconstrained free amplitude was
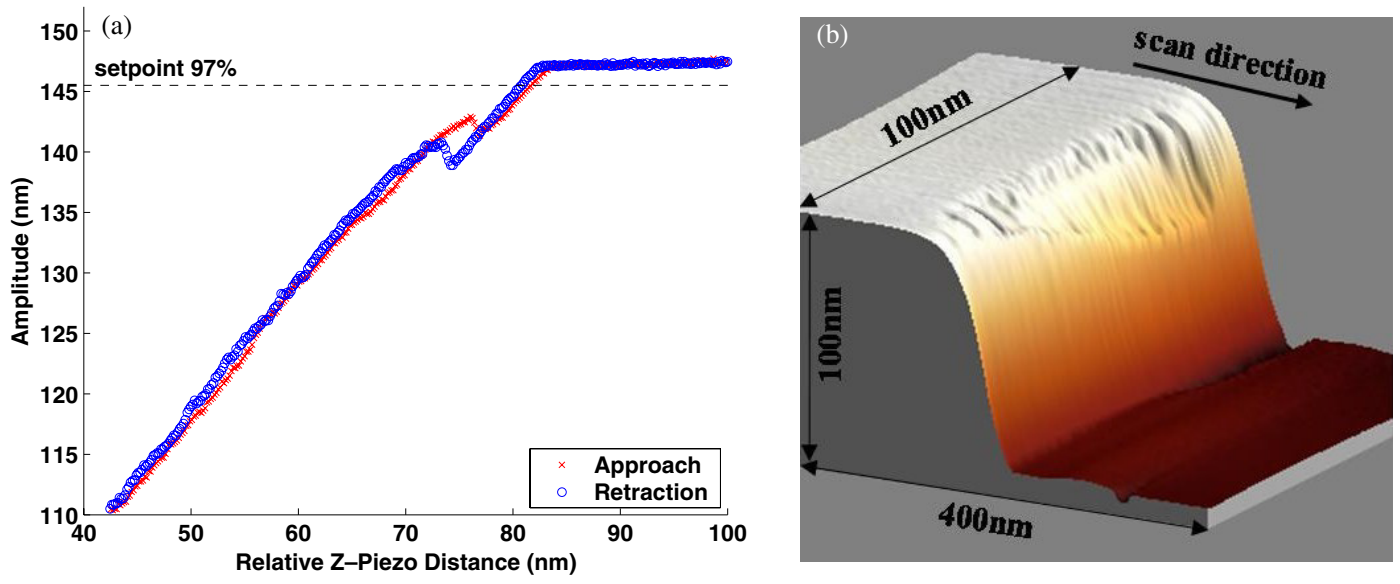

Figure 10. Unavoidable divot artefact seen on $\mathrm{SiO}_{2}$ trench wall when the unconstrained vibration amplitude of CNT probe I is very large at $147 \mathrm{~nm}$, even though the amplitude set-point ratio is very high at $97 \%$. 

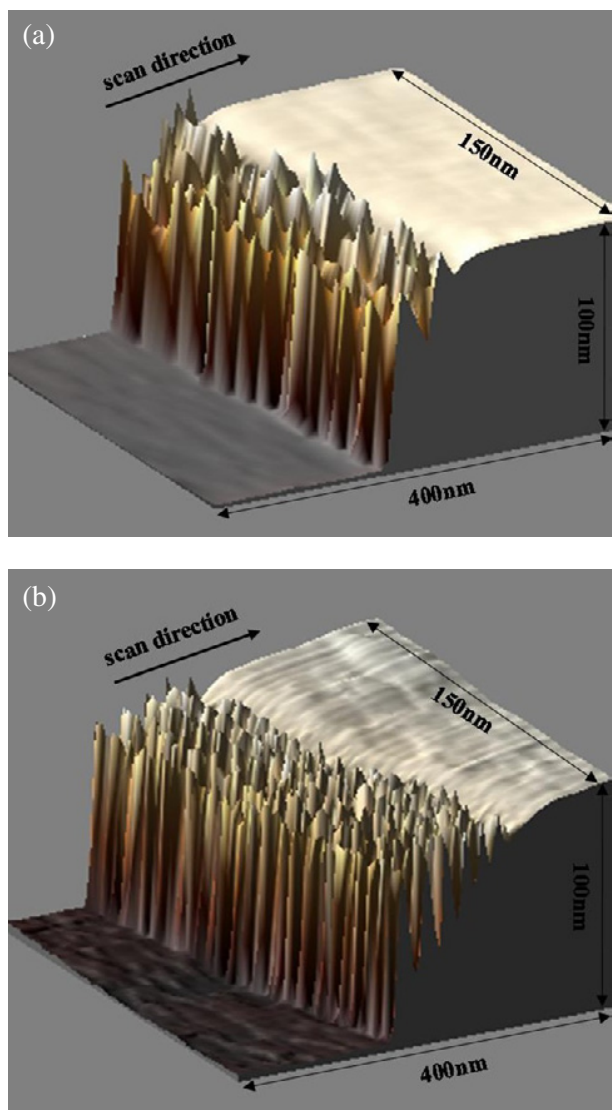

Figure 11. Ringing artefacts are shown near the bottom edge of a $\mathrm{SiO}_{2}$ trench wall while imaging with CNT probe I (a) and II (b) with respective unconstrained amplitudes of 19 and $105 \mathrm{~nm}$ and set-point amplitude ratios of $89 \%$ and $92 \%$.

lowered from 65 to $21 \mathrm{~nm}$. No ringing distortions were observed for unconstrained vibration amplitudes larger than $65 \mathrm{~nm}$.

The underlying cause of the ringing artefacts is CNT bending, adhesion, and stiction to the trench wall. Recall that the qualitative analysis of $[13,14]$ warned that SWCNTs could easily adhere to surfaces. The error signal in figure 12(c), which is the difference between the set-point amplitude and the actual amplitude, verifies that the ringing artefact is caused by the controller's reaction to CNT-trench wall adhesion and stiction. When the CNT probe initially encounters the edge at (a), the error signals for both the undistorted scan and the scan with the ringing artefact increase because of the topography change. However, the error signal peak at (b) for the ringing artefact scan is twice as large as the error signal for the undistorted image. The large error signal is due to the CNT probe-trench wall adhesion which reduces the vibrational amplitude of the cantilever. The controller perceives the sudden vibrational amplitude drop as a surface height increase, and responds by quickly moving the cantilever away from the sample. At point c, the CNT has finally broken free from the trench wall, and the cantilever, now very far from the sample, vibrates at its unconstrained vibration amplitude. In order to restore the original set-point amplitude ratio, the controller again lowers the probe. The error signal from $\mathrm{c}$ to $\mathrm{d}$ is flat because the probe was raised well above the attractive
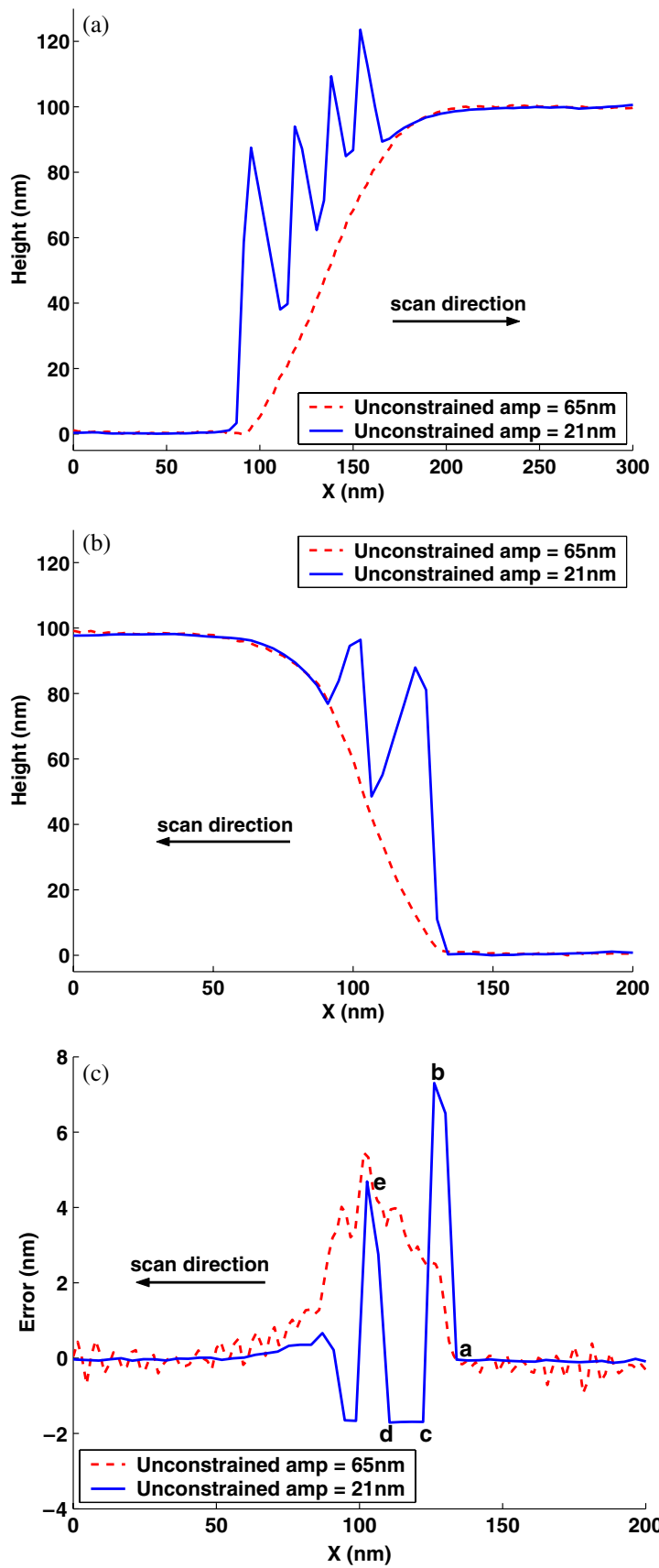

Figure 12. Line scans with $\mathrm{CNT} A \mathrm{AFM}$ probe $\mathrm{I}$ of $\mathrm{SiO}_{2}$ left and right trench walls show the ringing artefact when the unconstrained vibration amplitude was reduced from 65 to $21 \mathrm{~nm}$ while keeping the set-point amplitude ratio at $90 \%$. The CNT angle orientation as depicted in figure 6 causes a more pronounced ringing artefact for the right edge. The corresponding error signal for the left trench wall verifies that the artefact results from an interplay between CNT-trench wall stiction and controller reaction. (a) Right trench wall topography, (b) left trench wall topography, (c) left trench wall error signal.

regime before the CNT-trench wall stiction was overcome When the probe is lowered back into the attractive regime, the error signal begins to increase again at $\mathrm{d}$. As the probe is further lowered, the CNT again adheres to the trench wall, as shown by the peak at e. The process is then repeated to create the ringing artefact shown in figure 12(b). 
(a)
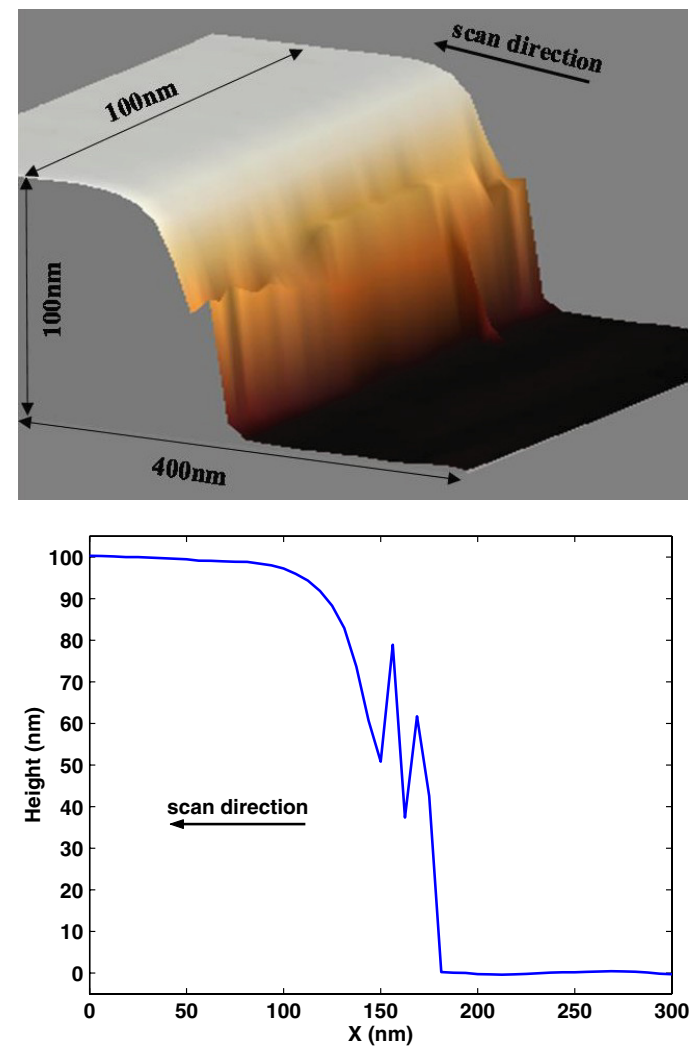

(b)
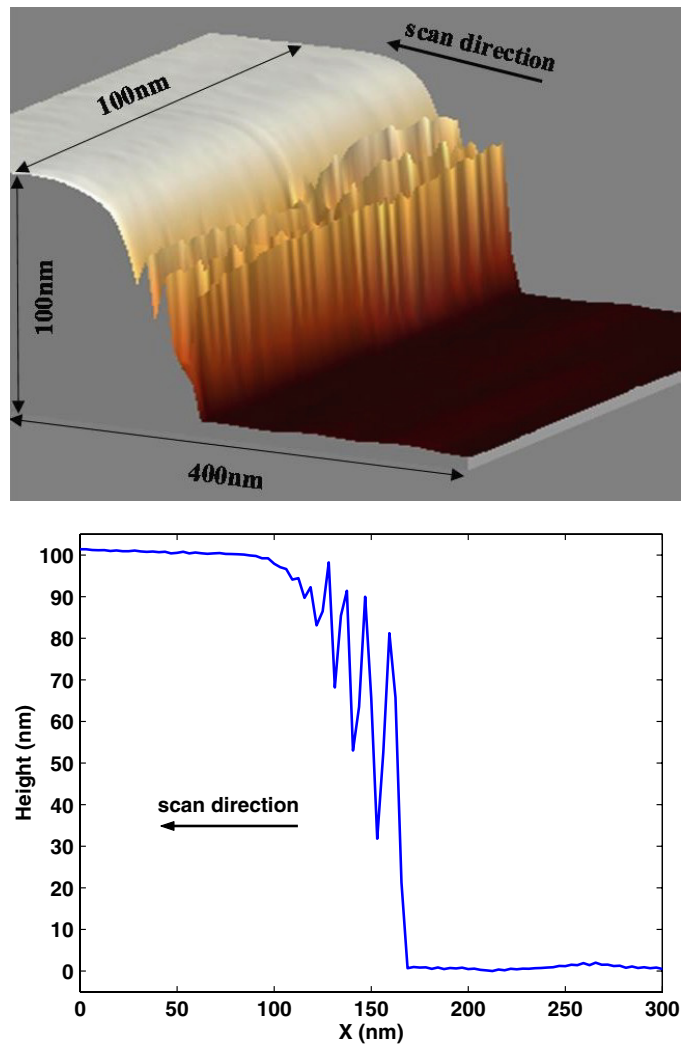

Figure 13. The three-dimensional topography and two-dimensional profiles of the $\mathrm{SiO}_{2}$ trench wall show that the ringing artefact is more pronounced when the set-point amplitude of CNT AFM probe I is lowered from (a) $96 \%$ to (b) $66 \%$ of the unconstrained vibration amplitude, which was kept at a constant $34 \mathrm{~nm}$.

As shown by figure 13, lower set-point amplitude ratios make the ringing artefact more pronounced, in that the frequency of ring oscillations increases, the height of each ringing oscillation is magnified, and the ringing effect occurs at higher points along the trench wall. CNT AFM probe I's setpoint amplitude was lowered from $96 \%$ to $66 \%$ of the $34 \mathrm{~nm}$ unconstrained vibration amplitude, as indicated by figure 14 . Smaller unconstrained vibrational amplitudes allow the CNT tip to spend more time, on average, near the trench wall edge, which increases the probability that the tip-trench wall lateral forces will be large enough to cause CNT bending, adhesion, and stiction.

Next, the existence of ringing artefacts is demonstrated for a different sample, the tungsten post array shown by the FESEM photograph in figure 15(a) and imaged with a conventional AFM probe in figure 15(b). Each post is $300 \mathrm{~nm}$ high with a $100 \mathrm{~nm}$ diameter, and is spaced in a grid with a $100 \mathrm{~nm}$ gap between nearby posts. Because the posts are tightly spaced, a conventional Nanosensors ${ }^{\mathrm{TM}}$ NCL AFM operating in the attractive regime is only able to obtain an absolute depth of $175 \mathrm{~nm}$. Figure 16, obtained with a CNT AFM probe, shows significant ringing artefacts near most edges of the post. The absolute depth measured with the CNT probe was no better than $200 \mathrm{~nm}$ because of ringing distortions from nearby posts. The ringing artefacts for the tungsten posts are especially prevalent compared to those for the $\mathrm{SiO}_{2}$ trench walls because the Hamaker constant relating graphite and tungsten is more than six times as large as that relating graphite and $\mathrm{SiO}_{2}[26,27]$. The ringing artefacts for the posts occur at greater distances from the edge because the $300 \mathrm{~nm}$ posts are three times as tall as $100 \mathrm{~nm} \mathrm{SiO}_{2}$ trench walls.

The asymmetric ringing artefacts of the tungsten posts which are most noticeable near the top and left edges are due to the CNT orientation angle relative to the post. In certain directions, the CNT orientation angle determines whether or not the ringing artefact occurs, as shown by the images of figure 17 scanned with the AFM microcantilever directed headon. When scanning head-on, the ringing artefact is only noticeable on the back edge, where the forward-aft CNT orientation angle displayed in figure 1(b) would typically reduce resolution. Figure 18 demonstrates that the ringing artefact is more pronounced on the edge with the more egregious angle distortion because geometrical effects make CNT bending and adhesion more probable.

\section{Correlation between DFM feedback parameters and image artefacts}

Both divot and ringing artefacts are influenced by scan speed, scan area, and integral gain. While divot artefacts are not as visible for large scan areas and fast scan speeds they cannot be averted when trying to carefully resolve high aspect ratio structures with scan areas less than $1 \mu \mathrm{m}$ wide. Similarly, as 

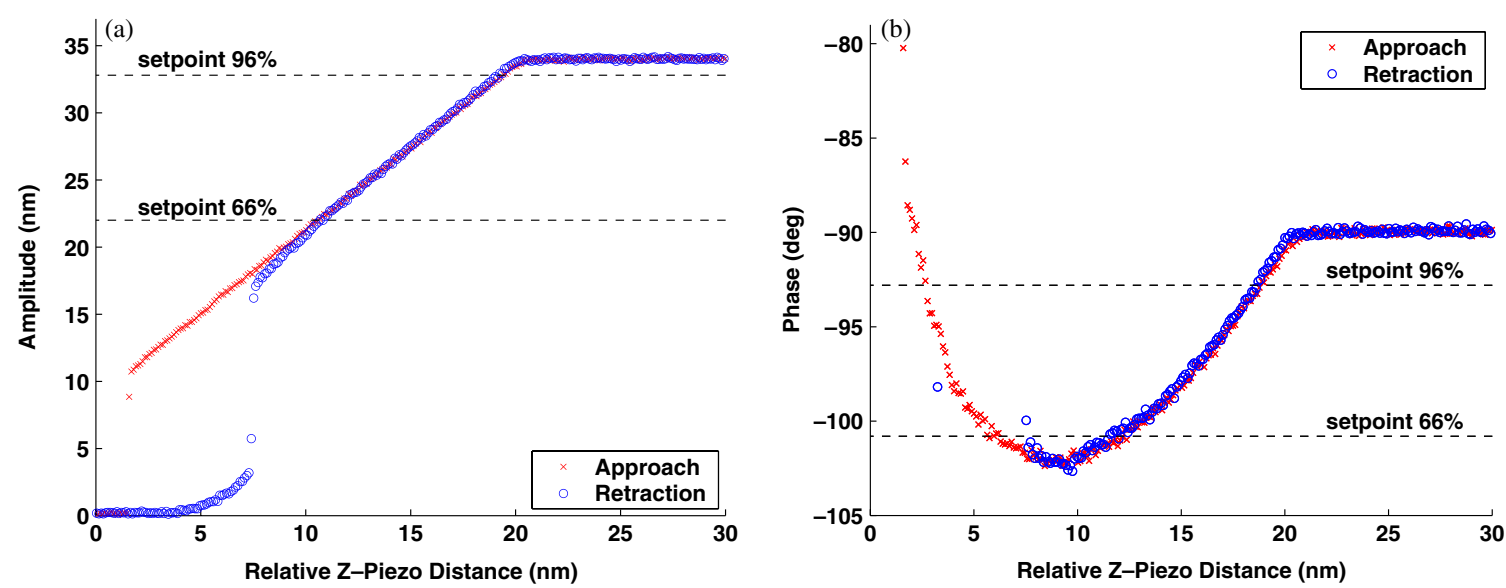

Figure 14. (a) Amplitude-distance and (b) phase-distance curves show the set-point amplitude ratios chosen before obtaining the ring artefact images seen in figure 13 with CNT probe I. The unconstrained vibration amplitude was kept at a constant $34 \mathrm{~nm}$.
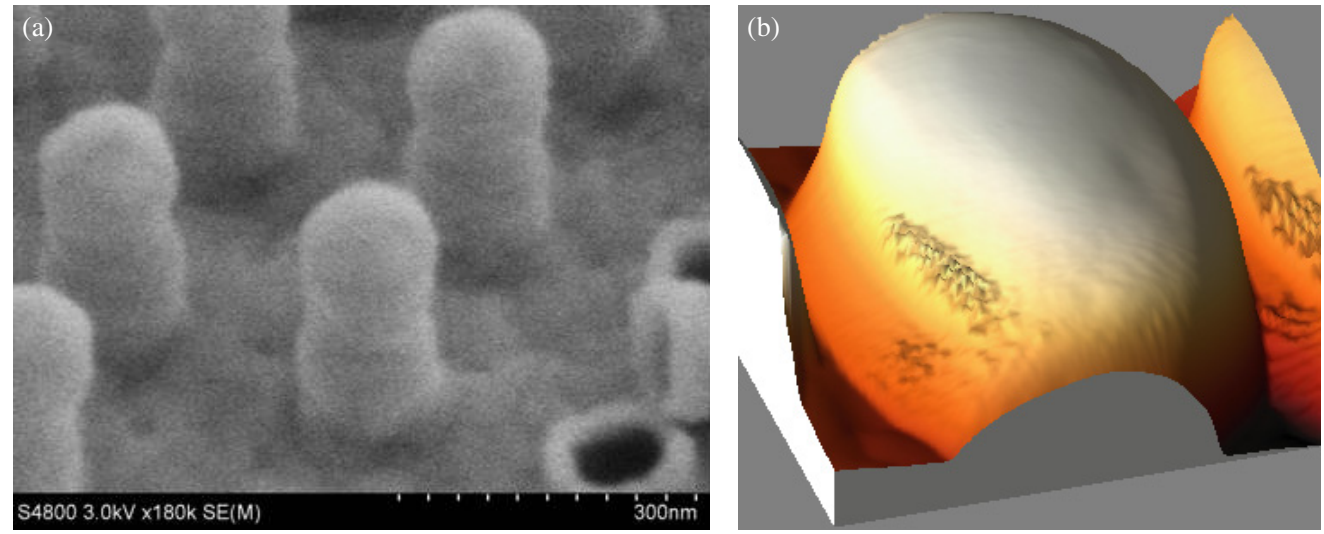

Figure 15. (a) FESEM image shows $300 \mathrm{~nm}$ high tungsten posts with $100 \mathrm{~nm}$ diameters and $200 \mathrm{~nm}$ distance between post centres, while the (b) conventional Nanosensor ${ }^{\mathrm{TM}}$ NCL AFM probe image of the posts is only able to measure a depth of $175 \mathrm{~nm}$.
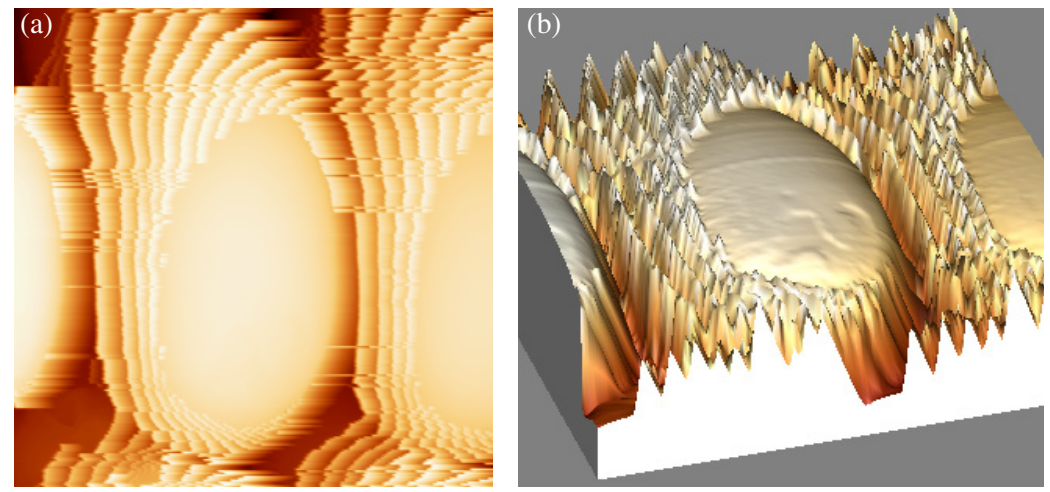

Figure 16. The two- and three-dimensional $600 \mathrm{~nm}$ square scans of tungsten posts imaged with CNT probe I operating with an unconstrained amplitude of $127 \mathrm{~nm}$ and an amplitude set-point ratio of $91 \%$ only record a depth of $200 \mathrm{~nm}$ between posts because of the ringing artefact caused by nearby posts.

long as the integral gain is large enough to properly resolve the high aspect ratio object, divot artefacts are a concern.

Although Park et al [18] suggested that slow speeds could eliminate the ringing artefact, we find that smaller scan areas and slower scan speeds only heightened the ringing artefact by giving the CNT more opportunity to adhere to the trench wall. Larger integral gains, which heighten the controller response time, also caused a more pronounced ringing artefact. As figure 19 demonstrates, increased integral gain exacerbates the ringing artefact because the controller reacts more quickly to 

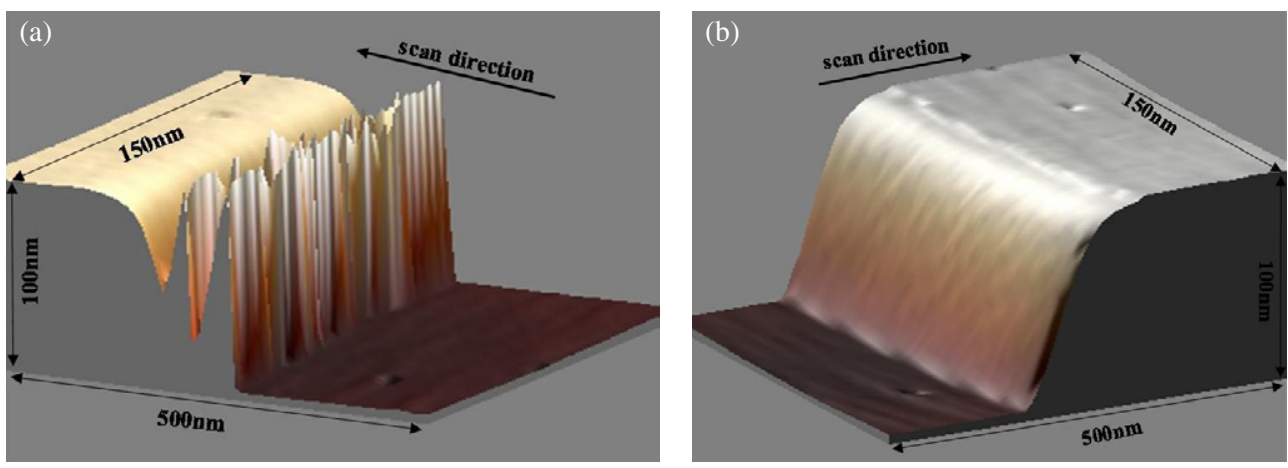

Figure 17. Attractive regime head-on scanned images of a $\mathrm{SiO}_{2}$ trench wall show that the ringing artefact only occurs on the back edge where the CNT orientation angle would normally cause a reduction in resolution. Images were obtained with CNT probe I with a unconstrained vibration amplitude of $61 \mathrm{~nm}$ and set-point amplitude ratio of 96\%. (a) Back edge (BE), (b) front edge (FE).

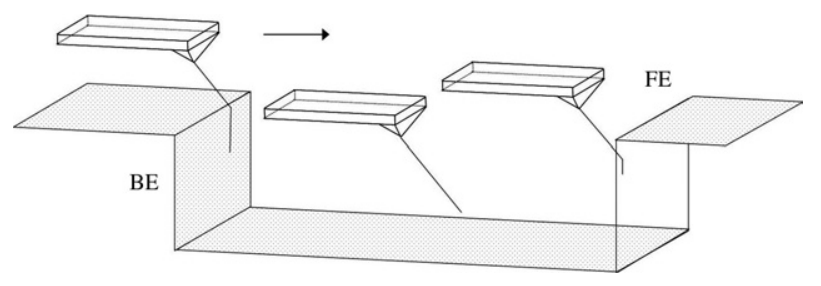

Figure 18. Schematic diagram demonstrating the head-on scan in which the AFM microcantilever's length is oriented perpendicular to the trench walls. The CNT orientation angle makes adhesion more likely on the back trench wall.

the CNT-trench wall adhesion and subsequent pull-off. When the integral gain is set to zero, figure 19 proves that a dulled ringing artefact still occurs, verifying that CNT-trench wall adhesion is the underlying cause of the distortion.

\section{Correlation between scan direction and image artefacts}

Both the divot and ringing artefacts are more distinct when ascending or hurtling over the trench wall as opposed to parachuting down from the top of it. This phenomenon, shown in figure 20 , was observed by scanning a $100 \mathrm{~nm} \mathrm{SiO}{ }_{2}$ trench wall in opposite directions. When hurtling, the probe continues scanning toward the trench wall while the controller simultaneously attempts to adjust the $Z$-distance. Because both artefacts depend on the CNT-trench wall interaction, the artefact becomes more pronounced as the probe continues to approach the trench wall. When parachuting, the probe is moving away from the trench wall, which provides less CNTtrench wall interaction time.

\section{Conclusions}

Carefully conducted experiments have shown that CNT probes, highly regarded for their high resolution imaging potential, are susceptible to imaging artefacts, particularly when imaging high aspect ratio structures with large sudden height changes like the $\mathrm{SiO}_{2}$ trench walls and tungsten posts. The angle, divot, and ringing artefacts have been observed and qualitatively explained by systematically changing the operating parameters

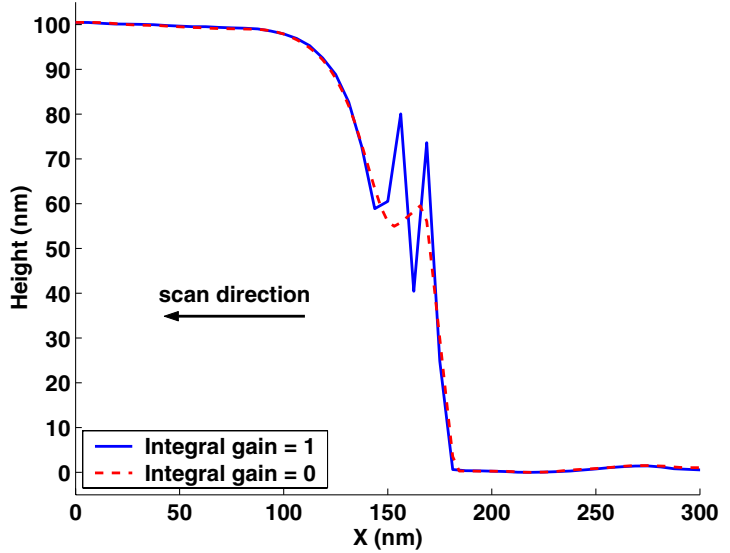

Figure 19. Profile scan of a $\mathrm{SiO}_{2}$ trench wall shows that the ring oscillations are less frequent and less amplified when the AFM controller gain is negligible, but significant enough to show that the underlying cause is due to the CNT itself. The scan was captured with CNT probe I operating with a $34 \mathrm{~nm}$ unconstrained vibration amplitude and $94 \%$ set-point amplitude ratio.

such as the amplitude set-point ratio, driving amplitude, integral gain, scan area, scan speed, and scan direction. Both the divot and ringing artefacts are caused by abrupt changes in the CNT-trench wall forces followed by the controller's reaction. Divot artefacts, observed near the tops of tall edges, occurred when the vertical forces between the CNT and sample were not large enough to sustain attractive regime imaging. A constant amplitude is maintained only when the CNT AFM probe begins imaging in the repulsive regime. Divot artefacts can be eliminated when the driving amplitude is small and the amplitude set-point ratio is kept high, though small driving amplitudes may make the CNT probe susceptible to ringing artefacts. Ringing artefacts were observed near the bottom of high aspect ratio structures and are caused by an interplay of CNT-trench wall stiction and controller reaction to the sudden force increase which it perceives as a topography change. Besides increasing the driving amplitude, ringing artefacts can be reduced by lowering the integral gain and by ensuring proper CNT alignment during the attachment process. 

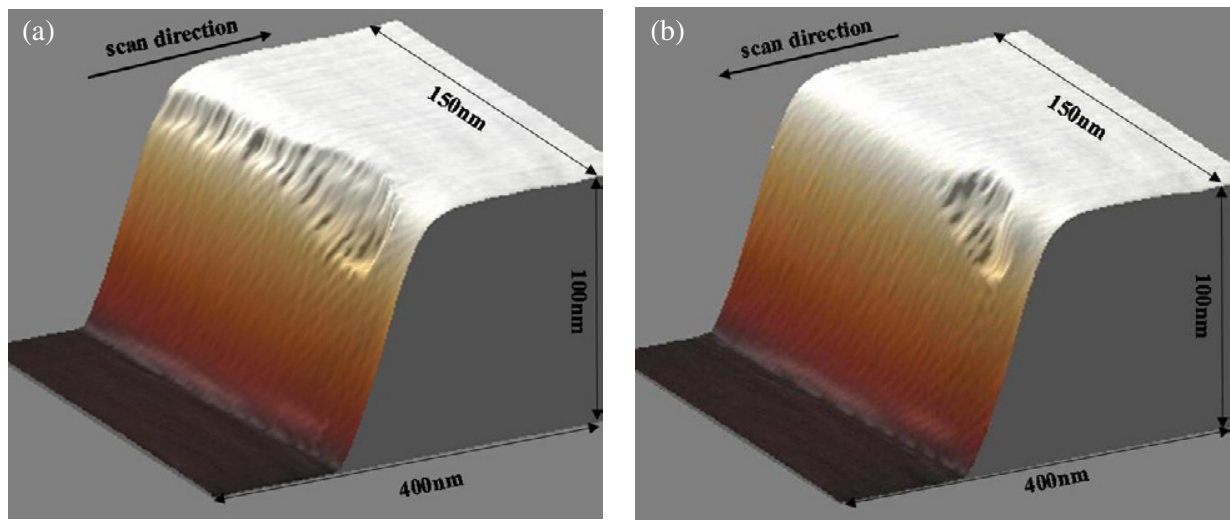

Figure 20. The $\mathrm{SiO}_{2}$ right trench wall images obtained with $\mathrm{CNT}$ probe I with a $69 \mathrm{~nm}$ unconstrained amplitude and amplitude set-point ratio of $81 \%$ show that the divot artefact is more pronounced when CNT probe I hurtles over the edge as opposed to parachuting down from the top of it. (a) Hurtling, (b) parachuting.

\section{Acknowledgments}

The authors would like to thank Hyung-Woo Lee at the Korea Advanced Institute of Science and Technology (KAIST) Mechanical Engineering Department in Taejon, Korea, for his work in creating CNT probes, and Ronald Reifenberger from the Physics Department at Purdue University in West Lafayette, Indiana, USA, for his valuable insight. Additionally, we are grateful for the financial support provided by the Centre for Nanoscale Mechatronics and Manufacturing in Korea and through the Winkleman Fellowship at Purdue University. Finally, C V Nguyen's contribution was supported through a subcontract from the University Affiliated Research Center at NASA Ames operated by the University of California Santa Cruz.

\section{References}

[1] Nguyen C V, Chao K-J, Stevens R M D, Delzeit L, Cassell A, Han J and Meyyappan M 2001 Nanotechnology 12363

[2] Bhushan B, Kasai T, Nguyen C V and Meyyappan M 2004 Microsyst. Technol. 10633

[3] Stevens R M D, Frederick N A, Smith B L, Morse D E, Stucky G D and Hansma P K 2000 Nanotechnology 11

[4] Larsen T and Moloni K 2002 Appl. Phys. Lett. 801996

[5] Yasutake M, Shirakawabe Y, Okawa T, Mizooka S and Nakayama Y 2002 Ultramicroscopy 9157

[6] Nguyen C V, Stevens R M D, Barber J, Han J, Meyyappan M, Sanchez M I, Larson C and Hinsberg W D 2002 Appl. Phys. Lett. 81901

[7] Hafner J H, Cheung C L, Woolley A T and Lieber C M 2001 Prog. Biophys. Mol. Biol. 7773
[8] Chen L, Cheung C L, Ashby P D and Lieber C M 2004 Nano Lett. 41725

[9] Luna M, de Pablo P J, Colchero J, Gomez-Herrero J, Baro A M, Tokumoto H and Jarvis S P 2003 Ultramicroscopy 9683

[10] Dietzel D, Faucher M, Iaia A, Aimé J P, Marsaudon S, Bonnot A M, Bouchiat V and Couturier G 2005 Nanotechnology 16 S73

[11] Lee S I, Howell S W, Raman A, Reifenberger R, Nguyen C V and Meyyappan M 2005 Ultramicroscopy 10395

[12] Barwich V et al 2000 Appl. Surf. Sci. 157269

[13] Snow E S, Campbell P M and Novak J P 2002 J. Vac. Sci. Technol. B 20822

[14] Snow E S, Campbell P M and Novak J P 2002 Appl. Phys. Lett. 802002

[15] Akita S, Nishikima H, Kishisa T and Nakayama Y 2000 Japan. J. Appl. Phys. 393724

[16] Akita S, Nishikima H and Nakayama Y 2000 J. Phys. D: Appl. Phys. 332673

[17] Kuwahara M, Hidekazu A, Tokumoto H, Shima T, Tominaga J and Fukuda H 2004 Mater. Charact. 5243

[18] Park B C, Kang J, Jung K Y, Song W Y, O B-h and Eom T B 2003 Proc. SPIE $\mathbf{5 0 3 8} 935$

[19] Lee H W, Kim S H, Kwak Y K and Han C S 2005 Rev. Sci. Instrum. 76046108

[20] Stevens R, Nguyen C, Cassell A, Delzeit L, Meyyappan M and Han J 2000 Appl. Phys. Lett. 773453

[21] Moloni K 2000 US Patent Application No 09/621, 710

[22] Moloni K, Lal A and Lagally M G 2000 Proc. SPIE 409876

[23] Lee S I, Howell S W, Raman A and Reifenberger R 2003 Ultramicroscopy 97185

[24] Schalf R et al 2002 Proc. SPIE 4689493

[25] Kühle A, Sørensen A H and Bohr J 1996 J. Appl. Phys. 81 6562

[26] Curran C, Lee J M and Watkins K G 2002 Opt. Laser Eng. 38 405

[27] Goodman F O and Garcia N 1991 Phys. Rev. B 434728 